\title{
ESTRUTURA E PROPRIEDADES DA DIFENIL-4-AMINA SULFONATO DE SÓDIO: UM DOPANTE DE FILMES CONDUTORES
}

\author{
José Maria Pires e Francisco Elias Jorge
}

Departamento de Física, Universidade Federal Espírito Santo, Av. Fernando Ferrari, 514, Campus de Goiabeiras, 29075-910 Vitória - ES, Brasil

Luiz Carlos Machado* e Antonio Augusto Lopes Marins

Departamento de Química, Universidade Federal Espírito Santo, Av. Fernando Ferrari, 514, Campus de Goiabeiras, 29075-910 Vitória - ES, Brasil

Recebido em 15/8/05; aceito em 6/1/06; publicado na web em 14/6/06

\begin{abstract}
STRUCTURE AND PROPERTIES OF DIPHENYL-4-AMINE SODIUM SULPHONATE: A DOPANT OF CONDUCTIVE FILMS. This work contains the theoretical simulation of the conformation of diphenyl-4-amine sodium sulphonate (DASNa) and correlates its geometry with conductivity, showing that the conductivity increases as the molecule becomes more planar. The solvent effect was also evaluated, using water and dimethylsulfoxide. Some properties, such as bond distance, vibration al frequency and effective charge were calculated. The utilization of diphenyl-4-amine sodium sulphonate (DASNa) as dopant of aniline was investigated in view of the HOMOLUMO energy gap.
\end{abstract}

Keywords: molecular modeling; polymers; solvent effect.

\section{INTRODUÇÃO}

Os compostos contendo sulfonatos desempenham funções importantes como aditivos de materiais da construção civil, como dopantes de polímeros orgânicos e no campo da catálise. A fabricação e o manuseio do cimento e concreto são facilitados pelos sulfonatos, aumentando o tempo de vida útil e a operacionalidade dos mesmos. Filmes anticorrosivos de polipirrol podem ser eletroquimicamente preparados contendo dopantes, como sulfonato de vinila e estireno sulfonato ${ }^{1-4}$. Produtos contendo sulfonato de sódio podem ser usados como aditivos emulsificadores e inibidores de corrosão $0^{5-7}$.

Em particular, a difenil-4-amina sulfonato de sódio, que denominaremos de DASNa, tem sido investigada em reações de homo e co-polimerização química com anilina, para formação de polímeros condutores solúveis em água ${ }^{8}$. A polianilina é um polímero orgânico estável ao ar, que apresenta propriedades condutoras úteis ao desenvolvimento de baterias, dispositivos eletrocrômicos ou, ainda, dispositivos microeletrônicos. O inconveniente da polianilina refere-se a sua limitada solubilidade em solventes orgânicos e em água, além da instabilidade em processos a altas temperaturas. A introdução de ácidos sulfônicos na cadeia da polianilina tornou a polianilina mais solúvel em água ${ }^{8,9}$.

Neste sentido, encontra-se registrada a reação de co-polimerização química da anilina dopada com DASNa para produzir a poli[anilinaco-N-(4-sulfofenil)anilina], um co-polímero solúvel em solução aquosa básica e com condutividade de $0,006 \mathrm{~S} \mathrm{~cm}^{-1}$. Da reação de homopolimerização entre os monômeros da anilina dopados quimicamente resulta a poli [N-(4-sulfofenil)anilina], com alto peso molecular e condutividade de $0,0035 \mathrm{~S} \mathrm{~cm}^{-1}$. Em ambas reações foi empregada como agente oxidante uma mistura de ácido clorídrico e persulfato de amônio. Os co-polímeros resultantes têm condutividades que variam entre a do homo-polímero e da polianilina $\left(5,2 \mathrm{~S} \mathrm{~cm}^{-1}\right)^{8,9}$.

Tem sido observada uma ampla gama de valores para as condutividades dos derivativos da polianilina, às vezes situadas no in-

*e-mail: luizcarlosmachado@bol.com.br tervalo $100-400 \mathrm{~S}$ e por outras entre $10^{-11}$ e $20 \mathrm{~S} \mathrm{~cm}^{-1}$, levantando dúvidas de que estes valores pudessem também resultar da elaboração experimental dos filmes ${ }^{10-12}$. Destaca também que dopantes introduzidos na polianilina, na posição orto, produziram copolímeros mais solúveis em água e com maior condutividade ${ }^{13}$. Mais recentemente foram estudados os efeitos de tamanho, densidade de carga, distribuição de carga na superfície dos dopantes, polarizabilidade e capacidade de oxidação dos ânions dopante p-tolueno sulfonato e cânfor sulfonato sobre a estrutura e as propriedades dos filmes de polianilina produzidos. Em particular, foi constatado que as distâncias entre as cadeias do polímero dependem do tamanho e da geometria dos dopantes. A decorrência prática dessas idéias é que a estrutura do filme é influenciada pela dimensão e forma geométrica dos dopantes adicionados aos monômeros durante a preparação dos filmes ${ }^{14}$. A compreensão das propriedades dos polímeros condutores, como a polianilina, tem sido bastante difícil de ser obtida e alguns pesquisadores sugerem que, para que se possa entender bem os vários fenômenos que ocorrem nesses polímeros, seria interessante o trabalho conjunto nas áreas de unificação de vários campos da física e química quântica molecular ${ }^{15}$.

Este trabalho procura associar propriedades moleculares, tais como estabilidade e reatividade à estrutura geométrica do composto dopante DASNa. Para tanto, obtivemos dados sobre as distâncias de ligação, distribuição de cargas e propriedades vibracionais. Foi também avaliada a influência dos solventes água $\left(\mathrm{H}_{2} \mathrm{O}\right)$, com constante dielétrica igual a 78,39 e dimetilsulfóxido (DMSO), com constante dielétrica igual a 46,7 na geometria da DASNa. Para analisar a condutividade da polianilina dopada com DASNa, foi construído um pequeno oligômero com três unidades e calculada a diferença de energia entre os níveis HOMO-LUMO.

\section{PARTE EXPERIMENTAL}

A geometria inicial usada como ponto de partida para otimização estrutural é apresentada na Figura 1a. A busca conformacional baseouse na variação dos ângulos diedro $\Phi_{1}$ (formado pelos átomos $24,1,2,4$ ) e $\Phi_{2}$ (formado pelos átomos $25,20,11,10$ ), iniciando-se com o valor 
igual a $0^{\circ}$ e promovendo sucessivas variações de $10^{\circ} \mathrm{em} 10^{\circ}$ até atingir o valor de $360^{\circ}$. Os valores do ângulo diedro $\Phi_{3}$ formado pelos átomos $(9,3,1,24)$ estão especificados na Tabela 2 . O ângulo entre os planos $(\sigma)$ é definido como sendo a diferença entre os ângulos $\Phi_{1}$ e $\Phi_{3}$

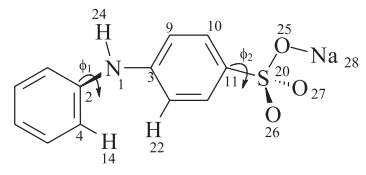

(a)

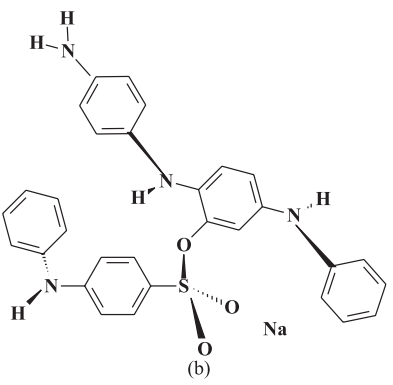

Figure 1. a) Molécula de difenil-4-amina sulfonato de sódio (DASNa); b) oligômero de polianilina dopada com a molécula de DASNa

Inicialmente, a molécula de DASNa foi construída com o Programa HyperChem 4.5 e otimizada com o campo de força MM+ ${ }^{16}$ (Figura 1). A seguir, foram efetuadas buscas conformacionais, empregando-se os métodos MM+, AM1 e PM3 e ab initio HF/3-21G*, com intuito de obter os mínimos de energia. Os confôrmeros de mais baixa energia foram inteiramente otimizados empregando-se os níveis de teoria acima, HF/6-21G* e B3LYP/6-31G*. Para realizar os cálculos semi-empíricos usamos os programas MOPAC 7.0 ${ }^{17}$, enquanto que para realizar os cálculo ab initio e DFT foi empregado o programa Gaussian $/ 03^{18}$. A análise conformacional para moléculas com muitos graus de liberdade é complexa ${ }^{19}$, entretanto no caso da DASNa, essa complexidade é bem menor, tendo em vista que podemos analisar o gráfico de energia potencial (GEP) levando em conta somente os ângulos torcionais $\Phi_{1}$ e $\Phi_{2}$. O modelo contínuo de solvatação PCM ("Polarizable Continuum Model") ${ }^{20}$ implementado no Programa Gaussian/03 foi usado para avaliar os efeitos dos solventes $\mathrm{H}_{2} \mathrm{O}$ e DMSO, sobre a molécula de DASNa. A diferença de energia entre os orbitais moleculares HOMO-LUMO foi avaliada usando-se métodos Hartree-Fock (HF) (cálculo realizado com as bases 3-21G* e 6$\left.31 \mathrm{G}^{*}\right)^{21-23}$ e a teoria do funcional da densidade (DFT). Nesta última aproximação, utilizamos o método híbrido de três parâmetros de Becke $^{24}$ juntamente com o funcional de correlação LYP ${ }^{25}$, originando o funcional extensivamente usado B3LYP.

Os cálculos foram realizados em um Pentium IV de 2,66 GHz e 512 MB de memória RAM, utilizando o sistema operacional Windows XP. As propriedades calculadas são apresentadas na Figura 2 e nas Tabelas 1 e 2.

\section{RESULTADOS E DISCUSSÃO}

As conformações moleculares caracterizam-se por um conjunto de variáveis geométricas, como comprimento, ângulo de ligações, ângulo diedro e interações não ligadas. Quando se utilizam campos de força em métodos de mecânica molecular deve-se selecionar e definir as contribuições mais adequadas, restringindo-se a cálculos de campos de força específicos que conservem as características do elemento químico coordenado ao ligante envolvido na ligação química ${ }^{26-30}$.

Aqui, foram examinadas as conformações da DASNa (métodos de mecânica molecular, semi-empíricos, ab initio e DFT), decorrentes das posições relativas entre os anéis benzênicos, e calculados os parâmetros geométricos associados aos confôrmeros, as propriedades eletrônicas, vibracionais, bem como a influência dos solventes $\mathrm{H}_{2} \mathrm{O}$ e DMSO sobre a molécula DASNa.
A rotação relativa entre os dois anéis benzênicos foi obtida indiretamente através da variação do ângulo de diedro $\Phi_{1}$ sendo o ângulo entre os dois anéis benzênicos $(\sigma)$ definido pelos átomos $4,2,3,9$. A escolha de um ângulo $\sigma$ igual a zero (ou $180^{\circ}$ ) irá implicar em admitir a co-planaridade entre os anéis. Entretanto, isto é impossível devido ao impedimento estéreo entre os dois hidrogênios (H14 e H22), que reduz a distância entre os átomos a 1,41 ̊. Pode ser verificado na simulação de mecânica molecular e $a b$ initio, através do gráfico da Figura 2, que para $\Phi_{1}$ próximo de $180^{\circ}$, o que corresponde à co-planaridade entre os anéis benzênicos, a energia atinge valores máximos. A Tabela 1 apresenta os valores otimizados dos ângulos $\Phi_{1}, \Phi_{3}$, o ângulo entre os dois anéis benzênicos $(\sigma)$ e as distâncias entre os hidrogênios H14 e H22 obtidas com os diferentes métodos. Para realizar os cálculos mostrados na Tabela 1, tomamos como ponto de partida a conformação em que $\Phi_{1}=140^{\circ}$ (que corresponde a $\sigma=120^{\circ}$ ) e $\Phi_{3}=20^{\circ}$. Ou seja, uma geometria inicial próxima de um dos mínimos encontrados através do cálculo PM3.

Na Figura 2 são mostrados os gráficos de energia potencial (GEP) decorrentes da análise conformacional realizada com os métodos $\mathrm{MM}+, \mathrm{AM} 1, \mathrm{PM} 3$ e ab initio $\mathrm{HF} / 3-21 \mathrm{G}^{*}$.
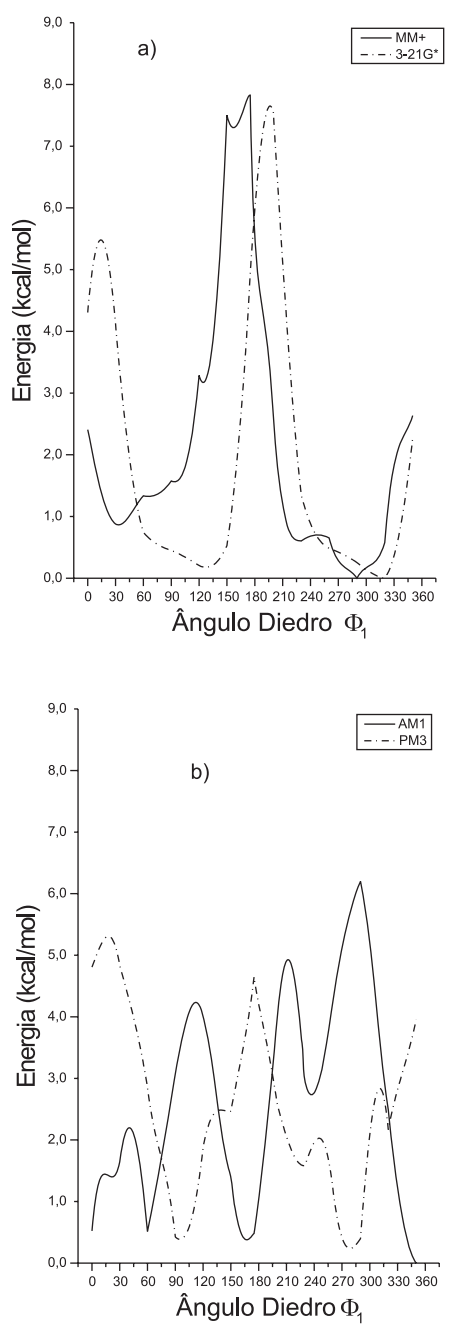

Figura 2. Gráfico da energia potencial (GEP) para a análise conformacional da rotação entre os anéis benzênicos $\left(\Phi_{1}\right)$, na molécula de DASNa. (a) Cálculos MM+e 3-21G*, ( b) cálculos AM1 e PM3

Através da análise dos gráficos da Figura 2, observa-se que o método AM1 apresenta uma região de mínimo entre $120^{\circ}$ e $210^{\circ}$, com mínimo bem pronunciado em $169,7^{\circ}$. Entretanto, o método PM3 apre- 
senta uma região de mínimo entre $30^{\circ}$ e $160^{\circ}$, detectando-se um pequeno mínimo em $146,8^{\circ}$. Vale ressaltar que a simulação através do uso de mecânica molecular com o campo de força MM+ (mais rápido e simples que qualquer método semi-empírico e $a b$ initio) apresentou um comportamento geral similar à simulação usando o método ab initio $\mathrm{HF} / 3-21 \mathrm{G}^{*}$, de forma que os mínimos de $\Phi_{1}$ encontrados nos dois casos são $150,4^{\circ}$ e $134,3^{\circ}$, respectivamente.

A rotação do grupo $\mathrm{SO}_{3} \mathrm{Na}$ em relação ao anel benzênico (representado na Figura 1a por $\Phi_{2}$ ) apresenta uma variação de energia da ordem da décima parte da variação $\Phi_{1}$. Observa-se também que $\Phi_{2}$ tem um mínimo absoluto em 89,6 (cálculo HF/3-21G*). Após analisar o conjunto de dados fornecidos pela busca conformacional mostrada nos gráficos da Figura 2, tomou-se como ponto de partida $\Phi_{1}$ $=140^{\circ}$ (que corresponde a $\sigma=120^{\circ}$ ) e, então, otimizou-se a molécula de DASNa em níveis HF/6-31G* e B3LYP/6-31G* obtendo-se $\Phi_{1}=145,4^{\circ}$ e $\Phi_{1}=152,3^{\circ}$, respectivamente.

Neste trabalho procurou-se investigar a conexão existente entre a posição relativa dos anéis benzênicos na molécula dopante DASNa, bem como a influência que esta exerce na distribuição eletrônica e na condutividade da polianilina. $\mathrm{O}$ monômero da anilina quando dopado e co-polimerizado com outra molécula de anilina produz a polianilina. Este procedimento é indicado para promoção de um aumento na solubilidade e na condutividade do polímero em água em relação às do monômero inicial ${ }^{13}$. Está claro que a DASNa foi inserida na molécula da anilina na expectativa de um aumento na condutividade do polímero, além da melhoria da solubilidade. Entende-se existir uma otimização da transferência de elétrons do aditivo para a molécula da anilina e desta para o oligômero, de maneira a estender o processo ${ }^{2,10,13}$. A não co-planaridade da DASNa se encontra ainda associada ao efeito estéreo dos volumosos grupos fenila, capazes de executarem movimentos torcionais importantes não só para a geometria do dopante como para as propriedades do oligômero constituinte do filme propriamente dito ${ }^{13}$.

Não menos importantes são os efeitos do solvente, sendo que a solubilidade dos filmes de polianilina em solventes orgânicos tem sido extensamente estudada mostrando a importância das propriedades ácido-base, da constante dielétrica e, ainda, do tamanho da molécula do solvente ${ }^{11}$. Neste trabalho o efeito dos solventes $\mathrm{H}_{2} \mathrm{O}$ e DMSO foi considerado implicitamente, usando-se o modelo PCM. Essa abordagem tem oferecido bons resultados na análise conformacional de moléculas como a tetraciclina, possibilitando estabelecer a influência do solvente sobre as propriedades da mesma ${ }^{31}$. Partindo dos cálculos ab initio e de DFT, no vácuo, apresentados na Tabela 1, reotimizou-se a molécula de DASNa, considerando implicitamente os solventes água e DMSO. Foi observado que a água torna a molécula mais plana. Em média, o ângulo entre os anéis benzênicos $(\sigma)$ diminui cerca de $3,2^{\circ}$, quando comparado com os valores da Tabela 1 . Ao se usar o solvente DMSO o ângulo entre os anéis benzênicos $(\sigma)$, diminui, em média, cerca de $3,0^{\circ}$ em relação aos cálculos realizados com o solvente $\mathrm{H}_{2} \mathrm{O}$. Acreditamos que esse ligeiro desvio no ângulo $\sigma$ torna a molécula mais condutora. Os resultados acima concordam com os dados experimentais em que são encontradas condutividades de $10^{-3} \mathrm{~S} / \mathrm{cm}$ e $10^{-2} \mathrm{~S} / \mathrm{cm}$ para os solventes $\mathrm{H}_{2} \mathrm{O}$ e $\mathrm{DMSO}^{8,11}$.

Com o objetivo de avaliar a influencia do dopante DASNa na condutividade da cadeia de polianilina, construímos um pequeno oligômero conforme mostra a Figura 1b. Esse modelo resulta de experimentos de eletro-polimerização da anilina na presença de dopantes moleculares do tipo sulfonatos ${ }^{32,33}$. Assim, o modelo de oligômero apresentando a interação da anilina com o dopante sulfonato através do átomo de oxigênio demonstrou mais consistência com os dados experimentais que aquele em que a interação privilegiava o átomo de enxofre. $\mathrm{O}$ balanço de carga no oligômero poderá ser mantido pela carga positiva (íon sódio), o qual também servirá como contra-íon ${ }^{33}$. A estrutura e as propriedades eletrônicas de pequenos oligômeros têm sido estudadas teoricamente, usando diferentes métodos semiempíricos, $a b$ initio e DFT. Sabe-se que a separação de energia molecular entre estados fundamental e excitado pode ser calculada, de maneira aproximada, como a diferença de energia HOMO-LUMO. A estimativa da separação de energia dos orbitais moleculares de fronteira apóia-se no teorema de Koopmans baseado no método $\mathrm{HF}^{34}$, incluindo aproximações semi-empíricas e métodos DFT ${ }^{35}$. No nível DFT, estes valores podem ser estimados diretamente a partir das equações de Kohn-Sham, contudo a precisão das propriedades previstas depende fortemente dos funcionais de troca e de correlação usados ${ }^{36}$. Deve-se ressaltar que De Oliveira e colaboradores ${ }^{37,38}$, utilizando o método ZINDO/S-CI para calcular separação de energia entre as bandas de valência e condução dos polímeros alquiltiofeno ${ }^{37} \mathrm{e}$ cianotiofeno ${ }^{38}$, obtiveram boa concordância com os dados experimentais. Uma vez que a DASNa não pode ser calculada com o método ZINDO/S-CI, pois o programa não dispõe de parâmetros para o átomo de sódio, nos restringimos a um cálculo HF/6-31G*. A condutividade dos oligômeros pode ser bem descrita experimentalmente pela diferença entre as energias da banda de valência $(B V)$ e de condução (BC), tomada aqui como sendo aproximadamente a diferença de energia entre os orbitais HOMO-LUMO.

Partimos da geometria obtida com os cálculos HF/6-31G*, descritos na Tabela 1 com o ângulo diedro $\Phi_{1}=145,4^{\circ}$. Após otimizar a geometria a nível HF/6-31G*, refizemos o cálculo acrescentando o dopante na posição orto. A partir desta geometria, realizamos cálculos a nível DFT com o funcional B3LYP. O oligômero puro apresentou uma diferença de energia entre os orbitais HOMO-LUMO de 3,21 eV, enquanto que no oligômero dopado com DASNa esse valor aumentou para $3,33 \mathrm{eV}$.

A inclusão do dopante piora a condutividade da polianilina ${ }^{8,9}$ passando de 5,2 para $3,510^{-3} \mathrm{~S} / \mathrm{cm}$. Em nosso cálculo de modelagem levou-se em conta somente o efeito do dopante DASNa, tratando-se de um sistema muito simplificado no qual a molécula encontra-se isolada e usa-se a diferença de energia entre os orbitais HOMO-LUMO para avaliar a condutividade do sistema. O resultado encontrado concorda com os dados experimentais ${ }^{8,9}$, uma vez que maior diferença de energia entre os orbitais HOMO- LUMO implica em menor condutividade.

A Tabela 1 contém os dados referentes a algumas distâncias de ligação da DASNa, calculadas através de campo de força (MM+), método semi-empírico (AM1 e PM3), método ab initio HF/3-21G* e HF/631G*, bem como B3LYP/6-31G*. Toda a molécula foi totalmente otimizada, após a análise conformacional, descrita anteriormente.

As distâncias S-O de 1,471 (método $a b$ initio HF/3-21G*) e de $1,468 \AA$ (método $a b$ initio $\mathrm{HF} / 6-31 \mathrm{G}^{*}$ ) para o oxigênio próximo ao

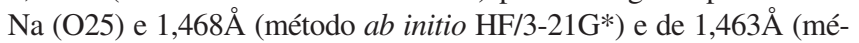
todo $a b$ initio $\left.\mathrm{HF} / 6-31 \mathrm{G}^{*}\right)$ para os oxigênios mais afastados $(\mathrm{O} 26$, O27) são as que mais se aproximam dos valores experimentais relatados para o grupo sulfonato no composto metilbenzenosulfonato de césio $^{39}$. Neste último composto, as distâncias S-O são, respectivamente, 1,452 1,442 e 1,441 A. Na DASNa as distâncias dos oxigênios O25, O26 e O27 ao Na são, respectivamente, 2,235 3,349 e 3,289 $\AA$ (método $a b$ initio HF/3-21G*) e 2,390 3,446 e 3,389 Å (método ab initio HF/6-31G*). O primeiro grupo de resultados (distâncias SO) mostra claramente a boa descrição das ligações iônicas da molécula pelos métodos ab initio, no entanto, o segundo grupo não permite uma comparação, a não ser intuitiva.

Os resultados obtidos nos cálculos $a b$ initio indicam que as distâncias C-N são significativamente diferentes entre si, ao contrário das demais aproximações, realçando a assimetria geométrica decorrente da presença do grupo sulfônico e do íon sódio no mesmo lado da molécula. Os resultados dos cálculos B3LYP/6-31G* (ver última coluna da Tabela 1) nos levam a crer que todos os valores são superes- 
Tabela 1. Distâncias de ligação $(\AA)$ e ângulos $\left(^{\circ}\right)$ para a DASNa, obtidas com alguns métodos teóricos

\begin{tabular}{lcccccc}
\hline $\begin{array}{l}\text { LigaçãoQuímica } \\
\text { e ângulos }\end{array}$ & MM+ & AM1 & PM3 & HF/3-21G* & HF/6-31G* & B3LYP/6-31G* \\
& $(\Delta)$ & $(\Delta)$ & $(\Delta)$ & $(\Delta)$ & $(\Delta)$ & $1,010(-0,011)$ \\
N1 - H24 & $1,021(-0,022)$ & $0,997(0,002)$ & $0,997(0,002)$ & $0,999(0,000)$ & $0,995(0,004)$ & $1,403(0,010)$ \\
N1 - C2 & $1,415(-0,002)$ & $1,403(0,010)$ & $1,440(-0,027)$ & $1,413(0,000)$ & $1,408(0,005)$ & $1,393(-0,009)$ \\
N1 - C3 & $1,414(-0,030)$ & $1,401(-0,017)$ & $1,445(-0,061)$ & $1,384(0,000)$ & $1,388(-0,004)$ & $1,779(-0,052)$ \\
S20- C11 & $1,716(0,011)$ & $1,719(0,008)$ & $1,814(-0,087)$ & $1,727(0,000)$ & $1,758(-0,031)$ & $1,506(-0,035)$ \\
S20- O25 & $1,640(-0,269)$ & $1,454(0,017)$ & $1,473(-0,002)$ & $1,471(0,000)$ & $1,468(0,003)$ & $1,501(-0,033)$ \\
S20- O26 & $1,559(-0,091)$ & $1,406(0,062)$ & $1,527(-0,059)$ & $1,468(0,000)$ & $1,463(0,005)$ & $1,005)$ \\
S20- O27 & $1,559(-0,091)$ & $1,405(0,063)$ & $1,521(-0,053)$ & $1,468(0,000)$ & $1,463(0,005)$ & $1,502(-0,034)$ \\
O25 - Na28 & $2,200(0,035)$ & $2,264(-0,029)$ & $2,582(-0,347)$ & $2,235(0,000)$ & $2,390(-0,155)$ & $2,412(-0,177)$ \\
H14 - H22 & $2,690(-0,171)$ & $2,243(0,276)$ & $2,245(0,274)$ & $2,519(0,000)$ & $2,564(-0,045)$ & $2,272(0,247)$ \\
$\Phi_{1}$ & $150,4^{\circ}\left(-16,1^{\circ}\right)$ & $169,7^{\circ}\left(-24,4^{\circ}\right)$ & $146,8^{\circ}\left(-12,5^{\circ}\right)$ & $134,3^{\circ}(0,00)$ & $145,4^{\circ}\left(-11,1^{\circ}\right)$ & $152,3^{\circ}\left(-18,0^{\circ}\right)$ \\
$\Phi_{3}$ & $19,2^{\circ}\left(-4,4^{\circ}\right)$ & $29,0^{\circ}\left(-14,2^{\circ}\right)$ & $21,9^{\circ}\left(-7,1^{\circ}\right)$ & $14,8^{\circ}(0,00)$ & $16,8^{\circ}\left(-2,0^{\circ}\right)$ & $17,8^{\circ}\left(-3,0^{\circ}\right)$ \\
$(\sigma)$ & $131,2^{\circ}(-11,7)$ & $140,7^{\circ}\left(-21,2^{\circ}\right)$ & $124,9^{\circ}\left(-5,4^{\circ}\right)$ & $119,5^{\circ}\left(0,00^{\circ}\right)$ & $128,6^{\circ}\left(-9,1^{\circ}\right)$ & $134,5^{\circ}\left(-15,0^{\circ}\right)$ \\
\hline
\end{tabular}

$\Delta$ - Diferenças entre as distâncias calculadas com os métodos MM+, AM1 e PM3, HF/6-31G* e B3LYP/6-31G*, em relação aos cálculos $a b$ initio usando-se a base $3-21 \mathrm{G}^{*}$.

timados em relação à verdadeira geometria da molécula. Deve-se notar que cálculos prévios de moléculas contendo elementos da primeira e segunda linhas da Tabela Periódica mostraram que os comprimentos de ligação geralmente aumentam quando os efeitos de correlação eletrônica são considerados ${ }^{40,41}$. A razão para isto pode ser simplesmente pensada como a participação de configurações de estados excitados, na formação de orbitais moleculares anti-ligantes.

Pelo conjunto total de dados apresentados nas Tabelas 1 e 2 (que será analisada em seguida), acreditamos que os cálculos ab initio $\mathrm{HF} /$ 3-21G* e HF/6-31G* (no que concerne às distâncias interatômicas) são os que melhor representam a verdadeira geometria da molécula de DASNa. Para ter uma idéia da variação dessas distâncias em função do método de cálculo empregado, tomamos por base o cálculo $a b$ initio $\mathrm{HF} / 3-21 \mathrm{G}^{*}$ e calculamos as diferenças desse valor para todos os outros $(\Delta)$. Os valores encontrados são apresentados entre parênteses na Tabela 1. Em geral, as propriedades como geometrias moleculares, barreiras de rotação interna, entalpias de formação e funções termodinâmicas podem ser reproduzidas por um campo de força simples $^{26-30}$. As simplificações introduzidas pelos métodos semi-empíricos (AM1 e PM3), que consideram apenas os elétrons de valência, conjuntos de base mínimos e parcialmente o recobrimento entre orbitais, tornam os cálculos mais rápidos, porém menos precisos ${ }^{42}$.

A Tabela 2 apresenta os valores escalonados ${ }^{43}$ calculados com os métodos semi-empíricos (AM1 e PM3), ab initio (HF/3-21G* e HF/631G*) e DFT (B3LYP/6-31G*) para as frequiências de vibração da molécula DASNa. Estes resultados foram comparados com os valores experimentais da ref. 44. Desta Tabela é evidente que os valores das freqüências vibracionais dependem da aproximação teórica utilizada. Em cálculos $a b$ initio, faz-se necessário incluir correlação eletrônica e trabalhar com um conjunto de base grande para se obter freqüências vibracionais em boa concordância com os respectivos valores experimentais ${ }^{45,46}$. Os erros percentuais médios entre os resultados obtidos com os modelos HF/3-21G*, HF/6-31G* e B3LYP/6-31G* e as correspondentes frequiências experimentais são 6, 8 e 9\%, respectivamente. Este resultado é surpreendente, visto que cálculos DFT levam em conta a correlação eletrônica. Constituem uma exceção os valores para as frequiências $\mathrm{C}=\mathrm{C}$ obtidas com o método semi-empírico PM3.

A complexa região compreendida entre as bandas localizadas entre 1130 e $1230 \mathrm{~cm}^{-1}$ do espectro experimental da DASNa (Tabela 2) refere-se às frequiências teóricas do grupo $\mathrm{SO}_{3} \mathrm{Na}$, as quais se apresentam bem descritas pela base $3-21 \mathrm{G}^{*}$. As freqüências obtidas com os métodos AM1 e PM3 nesta região são muito inferiores aos valores experimentais, devido à dificuldade de se encontrar uma parametrização adequada para o enxofre $\mathrm{f}^{46-48}$

Mesmo não sendo o objetivo principal deste trabalho, a alta intensidade da banda de absorção do estiramento $\mathrm{S}=\mathrm{O}\left(1170 \mathrm{~cm}^{-1}\right)$ do espectro experimental auxilia na escolha e diferenciação entre as várias estruturas conformacionais teoricamente possíveis ${ }^{46}$. O valor da frequiência de estiramento $\mathrm{N}-\mathrm{H}$ concorda com os dados da literatura ${ }^{45}$, onde os modelos AM1, PM3 e cálculos ab initio HF/6-31G e HF/6$31 \mathrm{G}^{*}$ foram empregados no estudo das freqüências de vibração da anilina e se mostraram coerentes em relação aos valores experimentais. Para as deformações angulares os erros são maiores e não acompanham a tendência anteriormente observada. Em geral, as frequiências das deformações angulares $\mathrm{C}-\mathrm{H}$ são superestimadas pelos cálculos AM1 e PM3 ${ }^{48,49}$. Por outro lado, as frequiências de estiramento C-H calculadas com estes métodos apresentam valores aceitáveis em relação aos valores experimentais ${ }^{45,48}$.

A ênfase na determinação das freqüências vibracionais está associada ao fato de que alguns sulfonatos, entre eles a DASNa, são usados como dopantes da anilina ${ }^{8,9,13}$. Tem sido prática comum examinar a dependência da forma das bandas contidas no espectro de IV do copolímero com a sua composição, variando-se a fração molar do sulfonato usado como aditivo ou dopante no co-polímero ${ }^{9,13}$. Para copolímeros da anilina dopados com ácidos alcoxisulfonados, como os ácidos aminopropanosulfônico e aminobutanosulfônico, um aumento da fração molar do aditivo poderá conduzir a aumento da intensidade de absorvância da vibração de estiramento $\mathrm{N}-\mathrm{H}$, vibração pouco intensa do grupo metileno ao redor de $1475 \mathrm{~cm}^{-1}$, forte incremento da intensidade do estiramento atribuído ao $\mathrm{SO}_{2}$ e $\mathrm{CO}_{2}$ em 1190 e 1045 $\mathrm{cm}^{-1}$, respectivamente, com significativa diminuição da banda de 830 $\mathrm{cm}^{-1}$ relativa à polianilina. Pode-se também notar que as bandas de 1500 e $1350 \mathrm{~cm}^{-1}$ diminuem com o decréscimo da fração molar da anilina, enquanto que as bandas de 1590 e $1640 \mathrm{~cm}^{-1}$ aumentam ${ }^{13}$.

Nos espectros de infravermelho apresentados na literatura e referentes ao produto da reação de co-polimerização química da anilina com a DASNa, ocorre a superposição de muitas bandas pertencentes a ambas substâncias (3400, 1510, 1590, 1340, 840 e $\left.745 \mathrm{~cm}^{-1}\right)$. Entretanto, bandas importantes, tais como as de 1045, 1190, 1173 e $1130 \mathrm{~cm}^{-1}$, associadas ao grupo sulfonato encontram-se disponíveis para acompanhamento da reação de co-polimerização ${ }^{13}$.

Dados de cargas efetivas (cálculo ab initio HF/3-21G*) e densidades eletrônicas apontam para uma deslocalização de elétrons na direção dos átomos de oxigênio do grupo sulfônico, com cargas efetivas no O25 $(-0,907)$, O26 (-0,902) e O27 (-0,711). O efeito é suficientemente forte para influenciar os valores das cargas efeti- 
Tabela 2. Freqüências experimentais e teóricas da DASNa em $\mathrm{cm}^{-1}$

\begin{tabular}{|c|c|c|c|c|c|c|}
\hline Atribuição & $\begin{array}{c}\text { AM1 } \\
(\text { Erro \%) }\end{array}$ & $\begin{array}{c}\text { PM3 } \\
(\text { Erro \%) }\end{array}$ & $\begin{array}{l}\mathrm{HF} / 321 \mathrm{G}^{*} \\
(\text { Erro \%) }\end{array}$ & $\begin{array}{l}\mathrm{HF} / 631 \mathrm{G}^{*} \\
(\text { Erro \%) }\end{array}$ & $\begin{array}{c}\text { B3LYP6-31G* } \\
\text { (Erro \%) }\end{array}$ & $\begin{array}{c}\text { Valores } \\
\text { Experimentais }\end{array}$ \\
\hline Fator de escala & 0,9532 & 0,9761 & 0,9085 & 0,8953 & 0,9614 & \\
\hline Deformação axial N-H & $3279(3,0)$ & $3266(3,4)$ & $3417(1,1)$ & $3440(1,8)$ & $3468(2,6)$ & 3381 (f) \\
\hline $\begin{array}{l}\text { Deformação axial } \\
\text { C-H Aromático }\end{array}$ & $3028(2,3)$ & $2974(4,1)$ & $3055(1,5)$ & $3021(2,6)$ & $3097(0,1)$ & 3100 (fr) \\
\hline Deformação angular N-H & $1520(4,6)$ & $1576(1,1)$ & $\begin{array}{l}1566(1,8) \\
1501(5,4)\end{array}$ & $\begin{array}{l}1510(5,3) \\
1482(6,6)\end{array}$ & $\begin{array}{l}1504(5,7) \\
1479(6,8)\end{array}$ & $\begin{array}{l}1594(\mathrm{f}) \\
1587(\mathrm{o})\end{array}$ \\
\hline Deformação axial $\mathrm{C}=\mathrm{C}$ & $\begin{array}{l}1697(12,2) \\
1660(11,3) \\
1659(13,6)\end{array}$ & $\begin{array}{l}1554(2,7) \\
1502(0,7) \\
1482(1,5)\end{array}$ & $\begin{array}{l}1456(3,8) \\
1402(6,0) \\
1352(7,4)\end{array}$ & $\begin{array}{c}1435(5,2) \\
1383(7,3) \\
1311(10,2)\end{array}$ & $\begin{array}{c}1435(5,2) \\
1387(7,1) \\
1352(12,3)\end{array}$ & $\begin{array}{l}1513(\mathrm{f}) \\
1492(\mathrm{v}) \\
1460(\mathrm{f})\end{array}$ \\
\hline $\begin{array}{l}\text { Deformação axial } \\
\text { C-N Aromático }\end{array}$ & $1384(1,1)$ & $1345(1,8)$ & $\begin{array}{l}1283(6,4) \\
1204(8,9)\end{array}$ & $\begin{array}{c}1270(7,3) \\
1175(11,1)\end{array}$ & $\begin{array}{l}1323(3,4) \\
1225(7,3)\end{array}$ & $\begin{array}{l}1370(\mathrm{v}) \\
1322(\mathrm{f})\end{array}$ \\
\hline Deformação axial $\mathrm{NaSO}_{3}$ & $\begin{array}{c}1022(16,9) \\
922(22,5) \\
784(33,2) \\
570(50,0) \\
510(48,8) \\
\end{array}$ & $\begin{array}{l}806(32,4) \\
773(35,1) \\
769(34,4) \\
502(56,0) \\
\\
\end{array}$ & $\begin{array}{c}1188(3,4) \\
1170(1,7) \\
1161(1,0) \\
1018(10,4) \\
1005(4,1) \\
590(14,9)\end{array}$ & $\begin{array}{c}1162(5,5) \\
1136(4,5) \\
1110(5,4) \\
1003(11,7) \\
975(7,0) \\
556(19,8)\end{array}$ & $\begin{array}{c}1118(9,1) \\
1095(8,0) \\
1093(6,8) \\
982(13,6) \\
947(9,6) \\
528(23,8)\end{array}$ & $\begin{array}{l}1230 \text { (f) } \\
1190 \text { (f) } \\
1173 \text { (f) } \\
1136 \text { (f) } \\
1048 \text { (f) } \\
693 \text { (v) }\end{array}$ \\
\hline $\begin{array}{l}\text { Deformação angular } \\
\text { C-H Aromático }\end{array}$ & $\begin{array}{l}951(13,1) \\
941(25,8) \\
850(20,2)\end{array}$ & $\begin{array}{l}951(13,1) \\
943(26,0) \\
821(16,1) \\
\end{array}$ & $\begin{array}{c}831(1,2) \\
787(5,2) \\
661(6,6) \\
538(26,3)\end{array}$ & $\begin{array}{l}803(4,5) \\
756(1,7) \\
633(10,5) \\
500(28,7)\end{array}$ & $\begin{array}{c}788(6,3) \\
736(1,6) \\
631(10,8) \\
490(33,0)\end{array}$ & $\begin{array}{l}841 \text { (f) } \\
748 \text { (f) } \\
707 \text { (f) } \\
730(v)\end{array}$ \\
\hline
\end{tabular}

Os fatores de escala usados nas freqüências foram obtidos na ref. 43 e encontram-se na linha onde-se lê: Fator de escala. Os valores experimentais foram obtidos da ref. 44. Os valores das intensidades experimentais são indicados a seguir: $(f=f o r t e)$, ( $f r=f r a c a),(v=$ variável), (o = ombro). O erro percentual (Erro \%) está indicado ao lado de cada freqüência, entre parênteses.

vas dos átomos de enxofre (20) $(1,717)$ e do carbono (11) $(-0,325)$ e está associado à indução exercida pelo cátion sódio.

\section{CONCLUSÕES}

As propriedades moleculares (estabilidade e reatividade) foram associadas à estrutura geométrica da molécula da DASNa, substância empregada como dopante de filmes orgânicos solúveis e condutores. Inicialmente estabeleceu-se a melhor posição relativa dos anéis benzênicos na DASNa, conectando-se a não co-planaridade do dopante e sua influência nas propriedades condutoras da polianilina. A geometria molecular inicial (ângulo entre os anéis benzênicos de $124^{\circ}$ ) serviu como ponto de partida para a busca conformacional. $\mathrm{O}$ ângulo de diedro $\Phi_{1}$ (formado pelos átomos $24,1,2,4$ ) foi alterado de $10 \mathrm{em}$ 10 graus, permitindo a rotação entre os dois anéis benzênicos e toda a molécula otimizada. Os cálculos ab initio HF/3-21G* mostraram um mínimo de energia para um ângulo entre os anéis igual a 119,5², levando-se a distribuição eletrônica não planar do sistema $\pi$. Desta forma, ficou evidenciado que as propriedades condutoras da polianilina dependem do valor do ângulo entre os anéis benzênicos da molécula da DASNa, sendo que os valores encontrados para estes ângulos, neste trabalho, são indicativos de severas restrições à transferência de elétrons.

A melhor geometria da DASNa parece ser descrita pelo cálculo $a b$ initio $\mathrm{HF} / 3-21 \mathrm{G}^{*}$, pois os resultados dos cálculos HF/3-21G* e HF/631G* mostram que as distâncias C2-N1 e C3-N1 são diferentes (Tabe- la 1), ao contrário das aproximações semi-empíricas, refletindo a presença do grupo sulfônico na molécula. Além disso, ao se consultar a Tabela 2 fica evidente que, de um modo geral, os melhores resultados obtidos para os valores das frequiências (com menor erro percentual, igual a 6\%) são aqueles fornecidos pelos cálculos HF/3-21G*.

A influência dos solventes água e dimetilsulfóxido sobre a molécula de DASNa foi avaliada através do modelo PCM, verificandose que a água torna a molécula ligeiramente mais co-planar que o vácuo $\left(3,2^{\circ}\right)$, enquanto que o dimetilsulfóxido aumenta um pouco mais essa co-planaridade $\left(3,0^{\circ}\right)$. No resultado final, o solvente dimetilsulfóxido torna a DASNa cerca de $6,2^{\circ}$ mais co-planar em relação ao vácuo.

Foi construído um pequeno oligômero de polianilina pura e dopada com DASNa. Calculou-se a diferença de energia entre os orbitais HOMO-LUMO encontrando-se, respectivamente, 3,21 e 3,33 eV, o que concorda com os dados experimentais.

Resumidamente podemos afirmar que a condutividade da polianilina (dopada com a molécula de DASNa) depende fortemente de vários fatores, tais como co-planaridade da molécula, deslocalização de elétrons em direção ao átomo de oxigênio do grupo sulfônico, efeito de diferentes solventes, diferença de energia entre os orbitais HOMO-LUMO e modo de preparação dos filmes.

As freqüências de vibração do grupo sulfônico são melhores descritas pelo método ab initio HF/3-21G*. Apesar da superposição nos espectros de infravermelho de bandas associadas ao produto da co-polimerização química da anilina e da DASNa, importantes ban- 
das atribuídas ao grupo sulfonato permitem o acompanhamento da reação de co-polimerização $\left(1045,1190,1173\right.$ e $\left.1130 \mathrm{~cm}^{-1}\right)$.

As cargas efetivas e as densidades eletrônicas nos átomos do composto, obtidas com o método ab initio, deram informações que contribuíram para localizar os possíveis sítios de ligação na molécula do dopante DASNa.

\section{AGRADECIMENTOS}

Ao Prof. Dr. A. C. Neto pelas discussões e sugestões feitas ao trabalho - DF - UFES. Ao Prof. Dr. S. O. Dantas pela cuidadosa leitura, discussão e sugestões feitas ao trabalho - DF - UFJF. Ao Prof. Dr. O. L. Alves pelo constante estímulo à pesquisa - IQ - Unicamp.

\section{REFERÊNCIAS}

1. Jeknavorian, A. A.; Mabud, Md.; Barry, E. F.; Litzau, J. J.; J. Anal. Appl. Pyrolysis 1998, 46, 8 .

2. Bhattacharya, A.; De, A. ; Das, S.; Polymer 1996, 37, 19.

3. Cao, G.; Mallouck, T. E.; Inorg. Chem. 1991, 14, 34.

4. Pinnavania, T. J.; Science 1983, 220, 365.

5. Eckardt, A.; Riff, I.; Weaver, J.; Lubr. Eng. 1997, 53, 17.

6. Mikulan, K. M.; Ross, N.; Lubr. Eng. 1999, 55, 21.

7. Hamdi, A. E. H.; Wedad, A. A. M.; Naguib, S. K.; Nader, A. G.; Ind. Eng. Chem. Res. 1993, 32, 1710.

8. Nguyen, M. T.; Kasai, P.; Miller, J. L.; Diaz, A. F.; Macromolecules 1994, 27,3625 .

9. De Armitt, C.; Armes, C. P.; Winter, J.; Uribe, F. A.; Gottesfeld, J.; Mombourquette, C.; Polymer 1993, 34, 158.

10. Furukawa,Y.; J. Phys.Chem. 1996, 100, 15644.

11. Cao, Y.; Qiu, P.; Synth. Met. 1995, 69,187.

12. Mac Diarmid, A. G.; Epstein, A. J.; Synth. Met. 1995, 69, 85.

13. Prevost, V.; Petit, A.; Pla, F.; Synth. Met. 1999, 104, 79.

14. Abd-Elwahed, A.; Holze, R.; Synth. Met. 2002, 131, 61.

15. Folch, S.; Régis, A.; Gruger, A.; Colomban, Ph.; Synth. Met. 2000, 110, 219.

16. Hypercube, Inc.; Programa Hyperchem 4.5; Gainesville, Estados Unidos da América, 1994.

17. Stewart, J. J. P.; Coolidge, M. B.; Danovich, D.; Klamt, A.; Danilov, V. I.; Kurtz, H.; Korambath, P.; Jensen, F.; Simmie, J. M.; Medrano, J. A.; Purvis III, G.; Danilov, V. I.; Frank J. Seiler Research Laboratory, U.S. Air Force Academy, Colorado Springs, CO 80840-6528, Estados Unidos da América, 1994.

18. Frisch, M. J.; Trucks, G. W.; Schlegel, H. B.; Scuseria, G. E.; Robb, M. A.; Cheeseman, J. R.; Montgomery, Jr., J. A.; Vreven, T.; Kudin, K. N.; Burant, J. C.; Millam, J. M.; Iyengar, S. S.; Tomasi, J.; Barone, V.; Mennucci, B.; Cossi, M.; Scalmani, G.; Rega, N.; Petersson, G. A.; Nakatsuji, H.; Hada, M.; Ehara, M.; Toyota, K.; Fukuda, R.; Hasegawa, J.; Ishida, M.; Nakajima, T.; Honda, Y.; Kitao, O.; Nakai, H.; Klene, M.; Li, X.; Knox, J. E.; Hratchian, H. P.; Cross, J. B.; Adamo, C.; Jaramillo, J.; Gomperts, R.; Stratmann, R. E.; Yazyev, O.; Austin, A. J.; Cammi, R.; Pomelli, C.; Ochterski, J. W.; Ayala, P. Y.; Morokuma, K.; Voth, G. A.; Salvador, P.; Dannenberg, J. J.; Zakrzewski,
V. G.; Dapprich, S.; Daniels, A. D.; Strain, M. C.; Farkas, O.; Malick, D. K.; Rabuck, A. D.; Raghavachari, K.; Foresman, J. B.; Ortiz, J. V.; Cui, Q.; Baboul, A. G.; Clifford, S.; Cioslowski, J.; Stefanov, B. B.; Liu, G.; Liashenko, A.; Piskorz, P.; Komaromi, I.; Martin, R. L.; Fox, D. J.; Keith, T.; Al-Laham, M. A.; Peng, C. Y.; Nanayakkara, A.; Challacombe, M.; Gill, P. M. W.; Johnson, B.; Chen, W.; Wong, M. W.; Gonzalez, C.; Pople, J. A.; Gaussian, Inc.; Pittsburgh PA, Estados Unidos da América, 2003.

19. Dos Santos, H. F.; Quim. Nova 2001, 24, 480.

20. Cossi, M.; Barone, V.; Cammi, R.; Chem. Phys. Lett. 1996, 255, 327

21. Wavefunction, Inc.; PC Spartan Pro; Irvine, Estados Unidos da América, 1999.

22. Dewar, M. J. S.; Zoebisch, E. G.; Stewart, J. J. P.; J. Am. Chem. Soc. 1985 , 107, 3902.

23. Hehre, W. J.; Radom, L.; Schleyer, P. V. R.; Pople, J. A.; Ab-initio Molecular Orbital Theory, John Wiley \& Sons: New York, 1986.

24. Becke, A. D.; J. Chem. Phys. 1993, 98, 5648.

25. Lee, C.; Yang, W.; Parr, R. G.; Phys. Rev. B.: Condens. Matter Mater. Phys. 1988, 37, 785

26. Coelho, L. W; Junqueira, G. M. A.; Herrera, J. O. M.; Machado, S. P.; Machado, B. C.; Quim. Nova 1999, 22, 396.

27. Comba, P.; Zimmer, M.; Inorg. Chem. 1994, 33, 5368.

28. Comba, P.; Bernhardt, P. V.; Inorg. Chem. 1992, 31, 2638.

29. Almeida, C. H. F.; Herrera, J. O. M.; Machado, S. P.; Quim. Nova 2002, 25, 957.

30. De Almeida, W. B; Quim. Nova 2000, 23, 600 .

31. Dos Santos, H. F.; Jr. Nascimento, C. S.; Belletato, P.; De Almeida, W. B.; J. Mol. Struc. (THEOCHEM) 2003, 626, 305.

32. Kilmartin, P. A.; Wright, G. A.; Synth. Met. 1997, 88, 153

33. Yue, J.; Epstein, A. J.; Synth. Met. 1995, 74, 123.

34. Koopmans, T.; Physica 1934, 1, 104

35. Levy, M.; Nagy, A.; Phys. Rev. A: At., Mol., Opt. Phys. 1999, 59, 1687.

36. Janak, J. F.; Phys. Rev. B: Condens. Matter Mater. Phys. 1978, 18, 7165.

37. De Oliveira, M. A.; De Almeida, W. B.; Dos Santos, H. F.; J. Braz. Chem. Soc. 2004, 15, 832 .

38. De Oliveira, M. A.; Dos Santos, H. F.; De Almeida, W. B.; Int. J. Quantum Chem. 2002, 90, 603.

39. Bo Sun, Y. Z.; Jin-Guang, W.; Quing-Chuan, Y.; Guang-Xian, X.; J. Mol. Struct. 1998, 470, 63.

40. Muniz, E. P.; Jorge, F. E.; Int. J. Quantum Chem. 2006, 106, 943.

41. Dykstra, C. E.; Schaefer, H.F.; The Chemistry of Ketenes and Allenes, John Wiley \& Sons: New York, 1980, cap. 1.

42. Sant'ana, C. M. R. ; de Souza, V. P. ; Quim. Nova 2001, 24, 583.

43. Scott, A. P.; Radom, L.; J. Phys. Chem. 1996, 100, 16502

44. Machado, L. C.; Marins, A. A. L.; Muri, E. J. B.; Lacerda, J. A. S.; Balthar, V. O.; Fulvio, P. F.; Freitas, J. C. C.; J. Therm. Anal. Calorim. 2004, 615.

45. Ramos, J. C. S.; Hollauer, E.; Cardoso, S. P.; Quim. Nova 1999, 22, 684.

46. Dos Santos, H. F.; De Almeida, W. B.; Do Val, A. M. G.; Guimarães, A. C.; Quim. Nova 1999, 22, 732

47. Seeger, D. M.; Korzeniewsky, C.; Kowalchyk, W.; J. Phys.Chem. 1991, 95,6871 .

48. Coolidge, M. B.; Martin, J. E., Stewart, J. J. P.; J. Comput. Chem. 1991, 12,948 .

49. Castellá-Ventura, M. Kassb, E.; Spectrochim. Acta, Part A 1994, 50, 69. 